\title{
The Thin Set Theorem for Pairs Implies DNR
}

\author{
Brian Rice
}

\begin{abstract}
Answering a question in the reverse mathematics of combinatorial principles, we prove that the thin set theorem for pairs (TS(2)) implies the diagonally noncomputable set principle (DNR) over the base axiom system $\mathrm{RCA}_{0}$.
\end{abstract}

\section{Background and Definitions}

The reverse mathematics of combinatorial principles has generated a good amount of interest in recent years. In particular, a lot of principles have emerged with reverse mathematical strength between $\mathrm{RCA}_{0}$ (recursive comprehension) and $\mathrm{ACA}_{0}$ (arithmetic comprehension) which are not equivalent to either of these axiom sets or to the

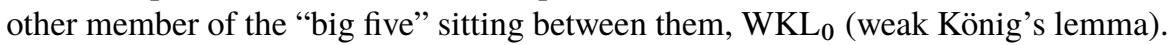
The picture in reverse mathematics has been greatly expanded by a large web of these combinatorial results, and it has been an ongoing and interesting project to understand this web, adding new principles and establishing implications (and nonimplications) among them.

Some illustrations and descriptions of this fascinating jungle can be found in Hirschfeldt and Shore [5] and Hirschfeldt, Shore, and Slaman [6]; here, we will be content with a brief outline of some of the more important bits of vegetation in order to appropriately locate the flora we are interested in examining.

We first remind the reader of a useful bit of notation: the symbol $[X]^{n}$ refers to the set of all unordered $n$-tuples whose elements are elements of the set $X$. Thus, a function $f:[X]^{n} \rightarrow \alpha$ should be interpreted as a coloring of the unordered $n$-tuples from $X$ with colors taken from the set $\alpha$.

1. Ramsey's theorem ( $\mathrm{RT}_{k}^{n}$ ). Ramsey's theorem is generally divided into a family of principles, referred to as $\mathrm{RT}_{k}^{n}$ for positive integers $n$ and $k$, and corresponding to $k$-colorings of $n$-tuples. In particular, $\mathrm{RT}_{k}^{n}$ states that for any function $f:[\omega]^{n} \rightarrow k$ there is an infinite homogeneous set $A$, that is, an

Received June 3, 2013; accepted June 24, 2013

2010 Mathematics Subject Classification: Primary 03B30; Secondary 03F35

Keywords: reverse mathematics, thin set, Ramsey's theorem

(C) 2015 by University of Notre Dame 10.1215/00294527-3153606 
infinite set $A \subseteq \omega$ such that $f$ restricted to domain $[A]^{n}$ takes only one value. It is easy to see that $\mathrm{RT}_{1}^{n}$ and $\mathrm{RT}_{k}^{1}$ are all provable in $\mathrm{RCA}_{0}$; moreover, it is also not too difficult to show by induction that $\mathrm{RT}_{k}^{n}$ and $\mathrm{RT}_{c}^{n}$ are equivalent for all $c, k \geq 2$. Not as obvious, but also known (due to Jockusch in [7]), is the fact that $\mathrm{RT}_{2}^{n}$ is equivalent over $\mathrm{RCA}_{0}$ to $\mathrm{ACA}_{0}$ for all $n \geq 3$. This leaves $\mathrm{RT}_{2}^{2}$ as the only remaining case.

2. Ramsey's theorem for pairs $\left(\mathrm{RT}_{2}^{2}\right)$. Ramsey's theorem for pairs lies strictly between $\mathrm{RCA}_{0}$ and $\mathrm{ACA}_{0}$ (the fact that it lies strictly below $\mathrm{ACA}_{0}$ is due to Seetapun and Slaman in [9]). It is known that $\mathrm{RT}_{2}^{2}$ neither implies nor is implied by $\mathrm{WKL}_{0}$ (the latter following from a result of Jockusch in [7] and the former due to Liu in [8]), and it is $\mathrm{RT}_{2}^{2}$ and principles weaker than it which make up much of the new jungle alluded to above.

3. Stable Ramsey's theorem for pairs ( $\mathrm{SRT}_{2}^{2}$ ). Stable Ramsey's theorem states that if $f:[\omega]^{2} \rightarrow 2$ is a function with the property that, for every $n$, there is some $M$ such that for all $m \geq M, f(\{n, m\})=f(\{n, M\})$ (this is sometimes stated as "for all $n, \lim _{m} f(\{n, m\})$ exists"), then there is an infinite homogeneous set $A$. The idea is that the coloring stabilizes; in the language of graphs, every vertex is connected either to cofinitely many other vertices by a color- 0 edge, or cofinitely many other vertices by a color- 1 edge. For a while it was an open question whether $\mathrm{SRT}_{2}^{2}$ was strictly weaker than $\mathrm{RT}_{2}^{2}$ or whether they were equivalent; this has been resolved by Chong, Slaman, and Yang [3] in favor of the former.

4. Cohesive principle $(\mathrm{COH})$. $\mathrm{COH}$ states that for any infinite sequence of sets $\left(R_{i}\right)_{i \in \omega}$, there is an infinite set $A$ such that for each $i$, either $A \subseteq \subseteq^{*} R_{i}$ or $A \subseteq \subseteq^{*} \overline{R_{i}}$. (Here, $\subseteq^{*}$ means that all but finitely many elements of the left-hand side are contained in the right-hand side.) As originally proved by Cholak, Jockusch, and Slaman in [2], $\mathrm{RT}_{2}^{2}$ is equivalent over $\mathrm{RCA}_{0}$ to the conjunction of $\mathrm{SRT}_{2}^{2}$ and $\mathrm{COH}$.

5. Chain-antichain, ascending or descending sequence, and friends (CAC, ADS, etc.). Chain-antichain (CAC) states that every infinite partial order has an infinite subset that is either a chain or an antichain. Ascending or descending sequence (ADS) states that every infinite linear order has either an infinite ascending sequence or an infinite descending sequence. These principles were explored by Hirschfeldt and Shore in [5], who showed that they lie strictly below $\mathrm{SRT}_{2}^{2}$. These principles themselves, like $\mathrm{RT}_{2}^{2}$, split into stable and cohesive versions: SCAC, CCAC (which is equivalent to ADS), SADS, and CADS. All of these splittings are strict.

6. Diagonally noncomputable set principle (DNR). A computability principle more measure-theoretic than combinatorial in flavor, DNR states that for every set $A$, there is a set which is diagonally noncomputable relative to $A$. DNR has proved to be important in that some, but not all, of the various combinatorial principles of interest imply it. For instance, $\mathrm{SRT}_{2}^{2}$ is known to imply DNR (proved by Hirschfeldt, Jockusch, Kjos-Hanssen, Lempp, and Slaman in [4]), but Hirschfeldt and Shore proved in [5] that CAC does not imply DNR, immediately giving the result that CAC does not imply $\mathrm{SRT}_{2}^{2}$. Other similar results have also been achieved.

7. Free set and thin set theorems $(\mathrm{FS}(n)$ and $\mathrm{TS}(n))$. The free set and thin set theorems represent another kind of weakening of Ramsey's theorem, dealing 
with colorings with infinitely many colors. The principle $\mathrm{FS}(n)$ states that for any function $f:[\omega]^{n} \rightarrow \omega$, there is an infinite free set $A$ such that $f(X) \in X \cup(\omega \backslash A)$ for every $n$-tuple $X$ of elements from $A$. The principle $\mathrm{TS}(n)$ states that for every such function, there is an infinite thin set $A$, with the property that $f$ restricted to $[A]^{n}$ omits some color; that is, $f\left([A]^{n}\right) \subsetneq \omega$. For every $n, \mathrm{RT}_{2}^{n}$ implies $\mathrm{FS}(n)$, which implies $\mathrm{TS}(n)$. (These facts, and others, are proved by Cholak, Giusto, Hirst, and Jockusch in [1] where they establish a number of results about free set and thin set.) These theorems are much weaker than Ramsey's theorem; while $\mathrm{RT}_{2}^{3}$ implies $\mathrm{ACA}_{0}$ already, Wang [10] has recently shown that even the conjunction of $\operatorname{FS}(n)$ (and $\operatorname{TS}(n))$ over all $n$ is not enough to imply $\mathrm{ACA}_{0}$.

The focus of this paper is on the thin set theorem for pairs, or TS(2). This principle should be regarded as a substantial weakening of $\mathrm{RT}_{2}^{2}$. $\mathrm{TS}(2)$ has something of a reputation for being almost uselessly weak; while it lies strictly above $\mathrm{RCA}_{0}$, it was known to imply almost nothing of note. The goal of this paper is to vindicate this little flower of the reverse math jungle and show that it is not, in fact, uselessly weak but represents a notably different direction of weakening of $\mathrm{RT}_{2}^{2}$ than the collection of principles living below CAC. Our goal is to prove the following theorem.

\section{Main Theorem $\quad \mathrm{RCA}_{0} \vdash \mathrm{TS}(2) \rightarrow \mathrm{DNR}$.}

We will approach the proof of the Main Theorem by first proving that it holds in $\omega$-models of $\mathrm{RCA}_{0}$ using techniques from computability theory, and then to show how to modify the proof to hold in the general setting. The interested reader may wish to compare this proof to the simpler proof that $\mathrm{SRT}_{2}^{2}$ implies DNR in [4] from which it takes its inspiration.

\section{$2 \omega$-Models of TS(2) Are Models of DNR}

Our proof that $\omega$-models of TS(2) are models of DNR begins with two lemmas.

Lemma 1 There are sets $\left\{A_{i}\right\}_{i \in \omega}$, uniformly $\leq_{T} 0^{\prime}$, a partition of $\omega$, and a computable function $f$, such that for every $e$, if $W_{e} \subseteq \overline{A_{i}}$, then $\left|W_{e}\right|<f(e, i)$. In fact we may take $f(e, i)=(k+1)(k+2)$, where $k=\max (e, i)$.

Proof Let $R(e, i)$ be the requirement "if $W_{e} \subseteq \overline{A_{i}}$, then $\left|W_{e}\right|<f(e, i)$."

We will prove this by $0^{\prime}$-computable construction in stages.

Begin with all $A_{i}$ empty. For each $n \geq 0$, at stage $n$, we have already decided in previous stages $y \in A_{i}$ (for any $i$ ) for at most $n(n+1)$ numbers (by induction) and will decide $y \in A_{i}$ for at most $2 n+2$ new numbers $y$ in stage $n$, giving at most $(n+1)(n+2)$ numbers in some $A_{i}$ (call these numbers used) by the end. We do this in three steps.

First, for each $0 \leq e<n$, we ensure that $R(e, n)$ is satisfied. Check using $0^{\prime}$ if $W_{e}$ has at least $(n+1)(n+2)$ elements. We are done with those $W_{e}$ which do not; they are small and $R(e, n)$ is already satisfied. For those which do, take one element from each which has not been used. Since only $n(n+1)$ numbers have been used, each such $W_{e}$ will contain such an element (in fact, $n$ such elements, so we can choose them all distinct if we like). Put all these elements into $A_{n}$, satisfying $R(e, n)$ by negating the hypothesis. This uses at most $n$ new numbers.

Next, we ensure that $R(n, i)$ is satisfied for each $0 \leq i \leq n$. Check if $W_{n}$ has at least $(n+1)(n+2)$ elements; if not, $R(n, i)$ is already satisfied for every $i$. If so, 
since we have used at most $n(n+1)+n$ numbers so far, $W_{n}$ contains at least $n+1$ elements which have not been used, say, $\left\{x_{i}\right\}_{0 \leq i \leq n}$. Put $x_{i} \in A_{i}$ for each $0 \leq i \leq n$, satisfying $R(n, i)$ for each $0 \leq i \leq n$. This uses at most $n+1$ new numbers.

Finally, if $n$ is not yet in some $A_{i}$, put $n \in A_{0}$.

Thus at the end of stage $n$, we have ensured that $R(e, i)$ is satisfied for each $0 \leq e, i \leq n$, used at most $n(n+1)+n+(n+1)+1=(n+1)(n+2)$ numbers total, and ensured that all numbers up to $n$ have been used.

The collection $\left\{A_{i}\right\}_{i \in \omega}$ constructed in the end are uniformly $0^{\prime}$-computable, since it takes only to stage $n$ to find out for which $A_{i}$ we have $n \in A_{i}$, and there is a unique such $A_{i}$, so this is a partition of $\omega$. Finally, all the requirements $R(e, i)$ are satisfied (each by stage $\max (e, i)$ ).

Observe that the proof, and thus the result, relativizes to $W_{e}^{X}$ and $\left\{A_{i}\right\}_{i \in \omega}$ uniformly $\leq_{t} X^{\prime}$.

Lemma 2 Let Turing ideal d model TS(2). Then for all $\left\{A_{i}\right\}_{i \in \omega}$ uniformly $\leq_{T} C^{\prime}$, where $C \in \mathcal{d}$, there is an infinite $B \in \mathcal{d}$ and an $n$ so that $B \subseteq \overline{A_{n}}$.

Proof By the limit lemma, there is a function $f: \omega^{2} \rightarrow \omega, f \leq_{T} C$ such that $A_{i}=\left\{x \in \omega: \lim _{m \rightarrow \infty} f(x, m)=i\right\}$. Taking $f:[\omega]^{2} \rightarrow \omega$ by ignoring $(x, m)$ with $x \geq m$ does not change these limits.

Then by TS(2), since $f \leq_{T} C \in \mathcal{d}$,

$$
\exists n \exists B \in \mathcal{Q}\left[f\left([B]^{2}\right) \subseteq \omega \backslash\{n\}\right] .
$$

So $\forall x \in B\left(\lim _{m \rightarrow \infty} f(x, m) \neq n\right)$, and hence $B \subseteq \overline{A_{n}}$.

Theorem 3 Every $\omega$-model of TS(2) is a model of DNR.

Proof Let $d$ be a Turing ideal that is an $\omega$-model of TS(2). We will show that $d$ contains a diagonally noncomputable function, but everything relativizes naturally to find a DNR relative to any $X \in \mathcal{\ell}$.

Let $\left\{A_{i}\right\}_{i \in \omega}$ be as in Lemma 1. By Lemma 2, there is an infinite $B \in \mathcal{d}$ and an $n \in \omega$ such that $B \subseteq \overline{A_{n}}$. Then for all $e$, if $W_{e} \subseteq B$, then $\left|W_{e}\right|<(k+1)(k+2)$, where $k=\max (e, n)$. Call this quantity $f_{n}(e)$.

Let $g$ be such that $W_{g(e)}$ is the set consisting of the first $f_{n}(e)$ many elements of $B$ ( $g$ is not computable, but is computable in $B$ ). For any $e$, if $W_{e}=W_{g(e)}$, then $W_{e} \subseteq B \subseteq \overline{A_{n}}$, and so $\left|W_{e}\right|<f_{n}(e)$ by construction of $A_{n}$. But $\left|W_{g(e)}\right|=f_{n}(e)$, which is a contradiction. So $\forall e\left(W_{e} \neq W_{g(e)}\right)$.

Now let $f$ be computable such that $W_{f(e)}=W_{\Phi_{e}(e)}$ if $\Phi_{e}(e) \downarrow$, and $W_{f(e)}=\emptyset$ otherwise, and consider $h=g \circ f$. Now if $\Phi_{e}(e) \downarrow$, then $W_{h(e)}=W_{g(f(e))} \neq$ $W_{f(e)}=W_{\Phi_{e}(e)}$, so it follows that $h(e) \neq \Phi_{e}(e)$. As $h$ is total (since both $f$ and $g$ are), this means that $h$ is a diagonally noncomputable function. But $h \leq_{T} B \in \mathcal{\ell}$, so $h$ is the function in $d$ that we wanted.

\section{TS(2) Implies DNR}

In order to check that $\mathrm{RCA}_{0} \vdash \mathrm{TS}(2) \rightarrow \mathrm{DNR}$, it suffices to show that the above proof can be carried out in $\mathrm{RCA}_{0}$. To do this we need to, first, eliminate all references to jumps (we can only talk about functions that exist in the model) and then check that the proof only requires $\Sigma_{1}^{0}$-induction. 
So, for instance, Lemma 1 becomes the following, recalling that there is an enumeration within a model $\mathcal{M}$ of all the $\mathcal{M}$-ce sets $W_{e}$ (where indices range over the first-order part of $\mathcal{M}$ ).

Lemma 4 There is a function $A(x, m)$ and a function $f(e, i)$ such that

(a) $\lim _{m} A(x, m)$ exists for each $x$,

(b) for each e, if $\forall x \in W_{e}\left(\lim _{m} A(x, m) \neq i\right)$, then $\left|W_{e}\right|<f(e, i)$.

Similarly, Lemma 2 becomes the following.

Lemma 5 For every function $A(x, m)$ such that $\lim _{m} A(x, m)$ exists for each $x$, there is an infinite $B$ and an $n$ such that $\forall x \in B\left[\lim _{m} A(x, m) \neq n\right]$.

The proof of Lemma 5 is exactly the same as that of Lemma 2, except it is even easier: there is no need to apply the limit lemma because we are using the limit notion in the first place as we do not have access to the jump. (Also, all instances of $\omega$ now refer instead to the first-order part of $\mathcal{M}$.)

The proof of Lemma 4 is somewhat more subtle, since we do not have access to any oracle with which to determine the size of $W_{e}$.

Proof Let $R(i, e)$ be the requirement that if $\forall x \in W_{e}\left(\lim _{m} A(x, m) \neq i\right)$, then $\left|W_{e}\right|<f(e, i)$. Let $\langle e, i\rangle$ be the pairing function that orders $\left(e_{1}, i_{1}\right)<\left(e_{2}, i_{2}\right)$ if $\max e_{1}, i_{1}<\max e_{2}, i_{2}$ or these are equal and $i_{1} e_{1}$ precedes $i_{2} e_{2}$ in lexicographic order. At stage 0 , we define $A(x, 0)=0$. At stage $m>0$, we do the following.

First, we run stages $0 \leq n<m$ from the proof of Lemma 1 as substages of stage $m$, except that, first, every time we need information about $W_{e}$ or its size, we use instead the corresponding information about $W_{e, m}$ (which we can know), and second, whenever we would put $x \in A_{i}$, we instead define $A(x, m)=i$. Also, omit the last step of each substage $n$ (where we would put $A(n, m)=0$ if it is not yet defined); it will not be necessary. Observe that for each $\langle e, i\rangle \leq\langle m, m\rangle$, this attempts to satisfy $R(e, i)$ (based on the assumption that $W_{e}=W_{e, m}$ ) in order. Say that the requirement $R(e, i)$ assigns $x$ at stage $m$.

Then, write $A(x, m)=0$ for all $x$ for which $A(x, m)$ is not yet defined.

There is a subtle problem here, in that in the original proof of Lemma 1, we on several occasions made an arbitrary choice from elements of $W_{e}$. If we happened to make different choices at different stages $m$ in the above construction, we could possibly ruin the existence of $\lim _{m} A(x, m)$. On the other hand, we cannot mandate that we always make the same choices, because if we chose an element from $W_{e_{1}}$, say, to put into $A_{i_{1}}$ (i.e., $A\left(x, m_{0}\right)=i_{1}$, using $x$ to satisfy $R\left(e_{1}, i_{1}\right)$ ), then discovered at a later stage $m_{0}$ that $W_{e_{2}}$ was large enough that it had to have an intersection with $A_{i_{2}}$, we might need to put $A\left(x, m_{1}\right)=i_{2}$. To solve this, we keep track of which requirement $R(e, i)$ assigns $x$ at stage $m$-and only this — to change to a higher priority requirement. Since we deal with requirements within each stage in their priority order, making this restriction does not hamper us at all.

We have to verify that, first, the construction can be carried out in $\mathrm{RCA}_{0}$, and second, $\mathrm{RCA}_{0}$ can verify that $\lim _{m} A(x, m)$ exists for all $x$ and satisfies the stated requirements.

It is clear that each of the things we wish to do can be carried out in $\mathrm{RCA}_{0}$ provided that we can show in $\mathrm{RCA}_{0}$ that, as in the proof of Lemma 1, when we begin substage $n$ of stage $m$ we have defined $A(x, m)$ for at most $n(n+1)$ many elements $x$. This can be shown by $\Delta_{1}^{0}$-induction (on $n$ ), so it holds in $\mathrm{RCA}_{0}$. 
Next, we verify that $\lim _{m} A(x, m)$ exists for each $x$. Suppose that there is a stage $m_{0}$ at which $A(x, m) \neq 0$ (if not, the limit exists and is 0 ). Then $x$ is assigned by some $R(e, i)$ at some stage $m_{0}$. Since $A\left(x, m_{1}\right)$ can only be defined differently if $x$ is assigned by a higher priority requirement at stage $m_{1}$ than at stage $m_{0}$, it follows that there are at most $\langle e, i\rangle+1$ many $m$ such that $A(x, m) \neq A(x, m+1)$. Hence $\lim _{m} A(x, m)$ exists.

Finally, we need to check that each $R(e, i)$ is satisfied. It follows by induction that for each $(e, i)$, there are at most $\langle e, i\rangle$ many numbers $x$ such that $A(x, m)$ is ever assigned by any requirement $R\left(e^{\prime}, i^{\prime}\right)$ with $\left\langle e^{\prime}, i^{\prime}\right\rangle<\langle e, i\rangle$. This is by $\Pi_{1}^{0}$-induction (which holds in $\mathrm{RCA}_{0}$ ), via a formula stating that for all finite sequences of length $\langle e, i\rangle+1$ and all $m$, it is not the case that each element of the sequence has been assigned by some $R\left(e^{\prime}, i^{\prime}\right)$ with $\left\langle e^{\prime}, i^{\prime}\right\rangle<\langle e, i\rangle$ by stage $m$. So there is some stage $m(e, i)$ after which no new elements are ever assigned by requirements $R\left(e^{\prime}, i^{\prime}\right)$ with $\left\langle e^{\prime}, i^{\prime}\right\rangle<\langle e, i\rangle$. So if $\left|W_{e}\right| \geq(k+1)(k+2)$, where $n=\max (e, i)$, then picking a stage $m \geq m(e, i)$ such that $\left|W_{e, m}\right| \geq(k+1)(k+2)$, we know that $A(x, m)=i$ for some $x \in W_{e, m}$, and furthermore, $x$ can never be assigned by some $R\left(e^{\prime}, i^{\prime}\right)$ with $\left\langle e^{\prime}, i^{\prime}\right\rangle<\langle e, i\rangle$. (It cannot have been before, since if it were, it could never have been assigned by $R(e, i)$ as which requirement assigns an element can change only to requirements of higher priority. And since it has never been, it cannot in the future by our definition of $m(e, i)$.) Hence, since $x$ is never assigned later by a requirement with higher priority, it will always be assigned by $R(e, i)$. Thus $A\left(x, m^{\prime}\right)=i$ for all $m^{\prime} \geq m ;$ that is, $\lim _{m}(x, m)=i$.

Since our two lemmas are both provable in $\mathrm{RCA}_{0}+\mathrm{TS}(2)$, it remains to check the construction of the function in the proof of Theorem 3. Everything in the construction goes through in $\mathrm{RCA}_{0}$ (pretty much verbatim, in fact), so we have the following.

Main Theorem $\quad \mathrm{RCA}_{0} \vdash \mathrm{TS}(2) \rightarrow \mathrm{DNR}$.

\section{Remarks}

It is worth noting that we do not require even the full strength of $\mathrm{TS}(2)$ for this proof. Even a "stable" version of TS(2) suffices, where we assume (naturally enough) that for each $x, \lim _{y} c(\{x, y\})$ exists (where $c$ is the coloring). This does not seem terribly interesting, however, if for no other reason than that the corresponding "cohesive" version of $\mathrm{TS}(2)$ is of course false.

The fact that TS(2) implies DNR but other weakenings of $\mathrm{RT}_{2}^{2}$, such as CAC, do not, suggests that DNR may prove to be a very useful tool for classifying various combinatorial principles. It has already been used to great effect to show several nonimplications among combinatorial principles, and the current result suggests that such use may be fruitfully expanded. It may be that DNR is a much more interesting point of comparison for the reverse mathematics of combinatorial principles than something like $\mathrm{WKL}_{0}$.

\section{References}

[1] Cholak, P. A., M. Giusto, J. L. Hirst, and C. G. Jockusch, Jr., "Free sets and reverse mathematics," pp. 104-19 in Reverse Mathematics 2001, vol. 21 of Lecture Notes in Logic, Association for Symbolic Logic, La Jolla, Calif., 2005. MR 2185429. 597 
[2] Cholak, P. A., C. G. Jockusch, Jr., and T. A. Slaman, "On the strength of Ramsey's theorem for pairs," Journal of Symbolic Logic, vol. 66 (2001), pp. 1-55. MR 1825173. DOI 10.2307/2694910. 596

[3] Chong, C. T., T. A. Slaman, and Y. Yang, " $\Pi_{1}^{1}$-conservation of combinatorial principles weaker than Ramsey's theorem for pairs," Advances in Mathematics, vol. 230 (2012), pp. 1060-77. MR 2921172. DOI 10.1016/j.aim.2012.02.025. 596

[4] Hirschfeldt, D. R., C. G. Jockusch, Jr., B. Kjos-Hanssen, S. Lempp, and T. A. Slaman, "The strength of some combinatorial principles related to Ramsey's theorem for pairs," pp. 143-61 in Computational Prospects of Infinity, Part II: Presented Talks, vol. 15 of Lecture Notes Series, Institute for Mathematical Sciences, National University of Singapore, World Science, Hackensack, N.J., 2008. MR 2449463. DOI 10.1142/9789812796554_0008. 596, 597

[5] Hirschfeldt, D. R., and R. A. Shore, "Combinatorial principles weaker than Ramsey's theorem for pairs," Journal of Symbolic Logic, vol. 72 (2007), pp. 171-206. MR 2298478. DOI 10.2178/js1/1174668391. 595, 596

[6] Hirschfeldt, D. R., R. A. Shore, and T. A. Slaman, "The atomic model theorem and type omitting," Transactions of the American Mathematical Society, vol. 361 (2009), pp. 5805-37. MR 2529915. DOI 10.1090/S0002-9947-09-04847-8. 595

[7] Jockusch, C. G., Jr., "Ramsey's theorem and recursion theory," Journal of Symbolic Logic, vol. 37 (1972), pp. 268-80. MR 0376319. 596

[8] Liu, J., "RT 2 does not imply WKL ${ }_{0}$," Journal of Symbolic Logic, vol. 77 (2012), pp. 609-20. MR 2963024. DOI 10.2178/js1/1333566640. 596

[9] Seetapun, D., and T. A. Slaman, "On the strength of Ramsey's theorem," Notre Dame Journal of Formal Logic, vol. 36 (1995), pp. 570-82. MR 1368468. DOI 10.1305/ndjfl/1040136917. 596

[10] Wang, W., "Some logically weak Ramseyan theorems," Advances in Mathematics, vol. 261 (2014), pp. 1-25. MR 3213294. DOI 10.1016/j.aim.2014.05.003. 597

Department of Mathematics and Computer Science

Huntington University

Huntington, Indiana, USA

brice@huntington.edu 